\title{
ESTUDIO SOBRE LOS NIVELES DE DEPENDENCIA EN PACIENTES ONCOLÓGICOS CON SINTOMATOLOGÍA RESPIRATORIA
}

\author{
STUDY ON THE LEVELS OF DEPENDENCE OF THE ONCOLOGICAL PATIENT WITH \\ RESPIRATORY SYMPTOMATOLOGY
}

\section{Eduardo José Fernández Rodríguez}

\begin{abstract}
RESUMEN
La incidencia del cáncer (Ca) en España está en aumento (247.771 nuevos diagnósticos). La Supervivencia de pacientes oncológicos a los 5 años es 53\%. Principal problema, es la calidad de esa supervivencia. Un Importante factor condicionante son los niveles de dependencia de los pacientes en tratamiento activo. Objetivo principal: Evaluar el nivel de dependencia de los pacientes oncológicos con disnea.

METODOLOGÍA: Estudio observacional prospectivo transversal con muestreo no probabilístico de asignación accidental. La muestra se reclutó del Servicio de Oncología Médica del Hospital Universitario de Salamanca. Se procedió a la derivación de los pacientes al programa de terapia ocupacional con la consiguiente inclusión de estos en el estudio. Posteriormentey tras obtener el consentimiento informado firmado, se procedió a evaluar. Se utilizó la escala de Lawton-Brody, y el índice de Barthel. Se diseñó una hoja de registro para datos sociodemográficos y clínicos. RESULTADOS: Muestra total de 180 individuos. Edad media de 68,48 ( $\pm 10,513)$ años. El 52,8\% varones, por un 47,2\% mujeres. El 22,8\% presentaban diagnóstico de Ca de mama, un 31,7\% Ca pulmonary un 45,6\% Ca relacionado con el aparato digestivo. En cuanto a los niveles de dependencia, escala de Lawton-Brody, puntuación media de 4,31 (₫1,832) puntos (niveles de dependencia moderados); índice de Barthel, puntuación media de 42,53 ( $\pm 2,789)$ puntos (nivel de dependencia moderado). Peores puntuaciones en diagnóstico anatomopatológico pulmonar. Correlación fuerte y estadísticamente significativa entre ABVD y AIVD $(r=0,803$, para $p<0,05)$, y correlación significativa $(r=-0,403$, para p<0,001) entre la edad y la realización de AIVD.

CONCLUSIÓN: Los pacientes oncológicos con disnea presentan unos niveles de dependencia moderada.
\end{abstract}

\section{PALABRAS CLAVE}

Nivel de dependencia, Actividades de la Vida Diaria, Cáncer, Terapia Ocupacional.

\begin{abstract}
The incidence of cancer ( $\mathrm{Ca}$ ) in Spain is increasing (247,771 new diagnoses). Survival of cancer patients at 5 years is $53 \%$. The main problem is the quality of that survival. An important conditioning factor is the level of dependence of patients in active treatment.

Aim: To evaluate the level of dependence of cancer patients with dyspnea.

METHODOLOGY: Cross-sectional prospective observational study with non-probabilistic accidental assignment sampling. Sample was recruited from the Service of Medical Oncology at the Salamanca University Hospital. The patients were referred to the occupational therapy program with their consequent inclusion in the study. Subsequently, and after obtaining signed informed consent, an evaluation was carried out. The Lawton-Brody scale and the Barthel index were used. A record sheet for sociodemographic and clinical data was designed.
\end{abstract}

1 Diplomado en Terapia Ocupacional en la Universidad de Salamanca, Máster en tratamiento de soporte y cuidados paliativos en la enfermedad oncológica, Máster en metodología de la investigación en ciencias de la salud y Programa Oficial de Doctorado en Oncología Clínica. Profesor asociado y cterapeuta ocupacional en el Servicio de Oncología Médica del Complejo Asistencial Universitario de Salamanca (AECC). Correo: edujfr@usal.es.ORCID 0000-0002-2175-8545 
RESULTS: Total sample of 180 individuals. Average age of 68.48 ( \pm 10.513 ) years. 52.8\% men, by $47.2 \%$ women. $22.8 \%$ had a diagnosis of breast $\mathrm{Ca}, 31.7 \%$ pulmonary Ca and $45.6 \%$ Ca related to the digestive system. Regarding the levels of dependency, Lawton-Brody scale, mean score of 4.31 ( $\pm 1,832)$ points (moderate levels of dependency); Barthel index, mean score of $42.53( \pm 2.789)$ points (moderate level of dependence). Worse scores in pulmonary pathological diagnosis.

Strong and statistically significant correlation between $A B V D$ and $A I V D(r=0.803$, for $p<0.05)$, and significant correlation ( $r=-0.403$, for $p$ $<0.001$ ) between age and AIVD performance.

CONCLUSION: Oncology patients with dyspnoea show moderate levels of dependence.

\section{KEYWORDS}

Level of dependency, Activities of Daily Life, Cancer, Occupational Therapy.

\section{INTRODUCCIÓN}

Analizados los datos presentes en el GLOBOCAN, actualmente la prevalencia en cáncer es de casi tres casos por cada mil habitantes en todo el planeta, siendo la segunda causa de muerte en el mundo (aproximadamente un $13 \%$ del total (National Comprehensive Cancer Network, 2018). Los tumores que más muertes causan cada año son los cánceres de pulmón, estómago, hígado, colon y mama (National Comprehensive Cancer Network, 2018).

Según la Sociedad Española de Oncología Médica, en los últimos datos publicados en 2014, se diagnostican 247.771 casos nuevos de cáncer cada año (148.827 en varones y 98.944 en mujeres) y más de 1,5 millones de personas están afectadas por la enfermedad.

El cáncer sigue constituyendo una de las principales causas de morbimortalidad, con aproximadamente 14 millones de casos nuevos en el mundo en el año 2018.

La mortalidad por cáncer en el año 2014 en España fue de 106.039 fallecimientos (65.019 casos en varones y 41.020 en mujeres).

A partir del cáncer o de sus tratamientos se producen en los individuos una serie de síntomas asociados que merman en estos, su capacidad para desenvolverse con normalidad en su vida cotidiana. Algunos de estos síntomas pueden ser la astenia, la ansiedad o la disnea. En esta última, las infecciones respiratorias constituyen una de las principales causas de muerte en el enfermo oncológico avanzado (Horneber, 2012). La disnea, junto con la tos y la hemoptisis, además de ser los síntomas más frecuentes en este tipo de pacientes, son los que más ansiedad producen tanto a las personas que lo padecen como a sus cuidadores (Fernández, 2020).

En algunas personas con Ca avanzado, esta disnea puede representar un signo clínico de la fase final de la enfermedad (American Occupational Therapy Association, 2015). Aproximadamente el $41 \%$ de las personas en cuidados paliativos tiene el síntoma y el $46 \%$ de ellas la describe de intensidad moderada o severa (Hewitt, 2003).

Al igual que en el caso de otro síntoma, como es el dolor, por ser una sensación subjetiva, sólo quien cursa la enfermedad puede juzgar de manera exacta la presencia y severidad de este.

Para el control de la misma, se consideró que no son suficientes las medidas empleadas en la práctica clínica convencional, sino que deben implementarse estrategias de tipo rehabilitador cuyo objetivo sea la reeducación del individuo en sus actividades de la vida diaria (AVD), no exclusivamente orientadas al manejo del síntoma, ya que la acción sobre la mejoría de los síntomas no consigue en la mayoría de los/las pacientes una mejora funcional de los mismos ni su readaptación a su actividad cotidiana (Fernández, 2018) .

La mayoría de los enfermos/as perciben esta sintomatología como un factor limitante fuera de su control que los lleva a realizar conductas de evitación que 
aumentan más su inactividad repercutiendo negativamente en su funcionalidad. Falta referencia que valide esta afirmación.

Es así como quienes presentan problemas respiratorios se adaptan a su sintomatología disminuyendo el nivel de actividad. Esto promueve un empeoramiento del estado físico y la disnea de esfuerzo. La disnea conlleva una incapacidad progresiva con pérdida de la movilidad, la autoestima, el trabajo y las relaciones sociales (Fernández, 2018).

Con estas referencias sobre las consecuencias de la patología respiratoria, se evidencia que disminuye la capacidad vital del individuo, provocando una limitación en su desempeño ocupacional. Debido a esto se consideró necesario plantear una intervención con perspectiva más rehabilitadora, e incorporación de profesionales de diferentes ámbitos de actuación sanitaria, incluyendo a los terapeutas ocupacionales, para conformar un equipo interdisciplinar.

En relación con los beneficios de la terapia ocupacional estos se apreciarán en (Sánchez, 2008):

- La calidad de vida y el bienestar general de la persona

- El estado general de salud, tanto relacionado con los síntomas respiratorios como en la tolerancia al ejercicio presente.

- La independencia del/la paciente que aumenta su autonomía y consecuentemente su sentimiento de autovalía.

- $\quad$ La habilidad para desempeñar sus roles ocupacionales en la sociedad.

En este marco, la actuación del terapeuta ocupacional siempre irá enfocada a cumplir todos los "Principios de los cuidados continuos y Paliativos" (Cooper, 2007).

- Atención integral del individuo.

- El enfermo/a y la familia son la unidad para tratar.

- Fomento de la autonomía y dignidad del enfermo/a.

- Concepción terapéutica activa y rehabilitadora.

- Objetivos de los cuidados: bienestar y calidad de vida.

- Importancia del ambiente o entorno.

Teniendo en cuenta los datos de incidencia y la mejora en términos de supervivencia en este tipo de patologías, creemos importante hacer hincapié en el control de esta sintomatología secundaria a estos procesos, por su repercusión en los niveles de dependencia de los individuos. Por ello, se plantea un estudio que verse sobre la evaluación de la sintomatología secundaria, haciendo hincapié en el grado de realización de actividades de la vida diaria, tanto las más básicas (autocuidado), como las instrumentales, puesto que la consecución de ambas será lo que determine de forma rigurosa su nivel de funcionalidad.

\section{Objetivo Principal}

Evaluar el nivel de dependencia de los/las pacientes oncológicos en tratamiento activo con sintomatología respiratoria.

\section{Objetivos Específicos}

- Determinar el nivel de realización de actividades básicas de la vida diaria (ABVD) en pacientes oncológicos en tratamiento activo con sintomatología respiratoria.

- Evaluar el nivel de realización de actividades instrumentales de la vida diaria (AIVD) en pacientes oncológicos en tratamiento activo.

- Analizar la repercusión del género en la realización de actividades instrumentales de la vida diaria de los pacientes con Ca en tratamiento activo con sintomatología respiratoria.

- Estudiar si existe relación entre la edad y la realización de actividades instrumentales de la vida diaria de los pacientes con Ca en tratamiento activo con sintomatología respiratoria.

- Establecer si existe diferencia en el grado de funcionalidad de los pacientes con Ca en tratamiento activo con sintomatología respiratoria.

\section{MÉTOdo}

DISEÑO: Para cumplir los objetivos se realizó un estudio observacional prospectivo transversal con muestreo no probabilístico de asignación accidental.

ÁMBITO DEL ESTUDIO: Servicio de oncología médica del Complejo Asistencial Universitario de Salamanca (CAUSA). 
POBLACIÓN Y ASIGNACIÓN MUESTRAL. La muestra constó de 180 pacientes procedentes del Servicio de Oncología Médica del Hospital Universitario de Salamanca y de la Asociación Española contra el Cancer (AECC), siendo reclutados para el estudio utilizando los siguientes criterios de inclusión y exclusión:

\section{Criterios de inclusión}

- Tener un diagnóstico anatomopatológico de enfermedad oncológica.

- Ser mayor de 18 años.

- Firmar el consentimiento informado mediante el cual autorizan su participación voluntaria en el estudio.

\section{Criterios de exclusión}

- Presentar un nivel de disnea inferior a 2 en la escala Medical Research Council (MRC).

\section{Criterios de retirada}

- No cumplimentar alguno de los registros autoadministrados.

- Retirarse voluntariamente del estudio aun habiendo firmado previamente el consentimiento informado.

PROCEDIMIENTO: Tras la inclusión de los/las pacientes en el programa de terapia ocupacional del servicio, se procedió a comprobar si las personas cumplían los criterios de inclusión para poder participar en el estudio. Posteriormente y tras obtener el consentimiento informado firmado, se procedió a la evaluación. Para la realización de esta se utilizó, por una parte, la escala de Lawton-Brody, y el índice de Barthel. Además, se diseñó una hoja de registro específica para la recogida de las diferentes variables a estudio.

\section{DESCRIPCIÓN DE LAS VARIABLES A ESTUDIO}

- Edad.

- Género.

- Diagnóstico anatomopatológico.

\section{INSTRUMENTOS DE EVALUACIÓN:}

1. Índice Barthel: Medida de la discapacidad física con demostrada validezy fiabilidad, fácil de aplicary de interpretary cuyo uso rutinario es recomendable. Útil para valorar la discapacidad funcional en las ABVD. Fiabilidad: Buena fiabilidad interobservador (índices de Kappa entre 0,47 y 1,00), fiabilidad intraobservador, (índices de Kappa entre 0,84 y 0,97) y consistencia interna (alfa de Cronbach de 0,86-0,92). Sensibilidad: Es capaz de detectar un progreso o deterioro en ciertos niveles del estado funcional, aunque su capacidad para detectar cambios en situaciones extremas es limitada.

2. Escala de Lawton y Brody: La escala de Lawton y Brody es uno de los instrumentos de medición de AIVD más utilizado internacionalmente y la más utilizada en las unidades de geriatría de España. Valora 8 ítems y les asigna un valor numérico 1 (independiente) o o (dependiente). La información se obtiene preguntando directamente al individuo o a su cuidador principal. Ha demostrado utilidad como método de valoración objetiva y breve que permite implantar y evaluar un plan terapéutico tanto a nivel de los cuidados diarios de los/las pacientes como a nivel docente e investigador. Escala muy útil para detectar las primeras señales de deterioro del anciano/a.

\section{Metodología Estadística}

\section{ESTADÍSTICA DESCRIPTIVA}

Las variables cuantitativas que siguen una distribución normal fueron definidas por media y desviación típica.

Para las variables cuantitativas que no siguen esta distribución, se utilizó la mediana en lugar de la media como medida de centralización.

Para evaluar si las variables siguen una distribución normal, se utilizó el test de Kolmogorov-Smirnoff.

Las variables cualitativas fueron definidas por número de casos y porcentajes. 


\section{ESTADÍSTICA ANALÍTICA}

Para analizar dos variables cuantitativas se va utilizó el coeficiente de correlación de Pearson.

Para evaluar dos variables cualitativas se utilizó la prueba estadística CHI-CUADRADO.

Para una variable cualitativa (2 categorías) y una variable cuantitativa, se usó la prueba estadística T- STUDENT.

Para definir una variable cualitativa (más de dos categorías) y una variable cuantitativa, se utilizó la prueba estadística ANOVA, cuya representación gráfica se lleva a cabo mediante diagramas.

\section{PROCESADO DE DATOS}

El proceso de análisis de datos se realizó con el programa estadístico SPSS versión 24.0

\section{Resultados}

Los resultados correspondientes a la parte descriptiva del estudio de las diferentes variables analizadas se pueden observar en las Tabla 1 y 2. Si los analizamos se observa que la muestra total del estudio fue de 180 individuos. La edad media de la muestra fue de 68,48 $( \pm 10,513)$ años. En cuanto al género de los participantes, el $52,8 \%$ de la muestra fueron varones, por un $47,2 \%$ mujeres (85 mujeres y 95 hombres). Respecto al diagnóstico anatomopatológico el 22,8\% presentaban diagnóstico de mama, un $31,7 \%$ pulmonar y un $45,6 \%$ presentaba tumores relacionados con el aparato digestivo.

TABLA 1 ANÁLISIS DESCRIPTIVO DE LAS VARIABLES INTERVINIENTES SEXO Y EDAD A ESTUDIO

\begin{tabular}{c|c|c|c}
\hline \multicolumn{2}{c|}{ SEXO } & \multicolumn{2}{c}{ EDAD } \\
\hline \multicolumn{2}{c|}{$\mathrm{N}(180)$} & Media & S \\
\hline Varón & $95(52,8 \%)$ & & \\
Mujer & $85(47,2 \%)$ & 68,48 & 10,513 \\
\hline
\end{tabular}

Tabla 1. Resultados descriptivos variables intervinientes

TABLA 2 ANÁLISIS DESCRIPTIVO DE LAS VARIABLES INTERVINIENTES DIAGNÓSTICO ANATOMOPATOLÓGICO A ESTUDIO

\begin{tabular}{|c|c|c|c|c|c|}
\hline \multicolumn{6}{|c|}{ DIAGNÓSTICO ANATOMOPATOLÓGICO } \\
\hline \multirow{2}{*}{ Mama } & \multirow{N}{*}{$\mathrm{N}=41$} & \multirow{2}{*}{$22,8 \%$} & Varón & 0 & $0 \%$ \\
\cline { 4 - 6 } & & & Mujer & 41 & $100 \%$ \\
\hline \multirow{2}{*}{ Pulmonar } & \multirow{2}{*}{$\mathrm{N}=57$} & \multirow{2}{*}{$31,7 \%$} & Varón & 41 & $71,92 \%$ \\
\cline { 4 - 6 } & & & Mujer & 16 & $28,07 \%$ \\
\hline \multirow{2}{*}{ A. Digestivo } & \multirow{2}{*}{$\mathrm{N}=82$} & \multirow{2}{*}{$45,6 \%$} & Varón & 54 & $65,85 \%$ \\
\cline { 4 - 6 } & & & Mujer & 28 & $34,14 \%$ \\
\hline
\end{tabular}

Tabla 2. Distribución por diagnóstico anatomopatológico y por sexo. 
FIGURA 1 GRÁFICO DE LA VARIABLE INTERVINIENTE "SEXO"

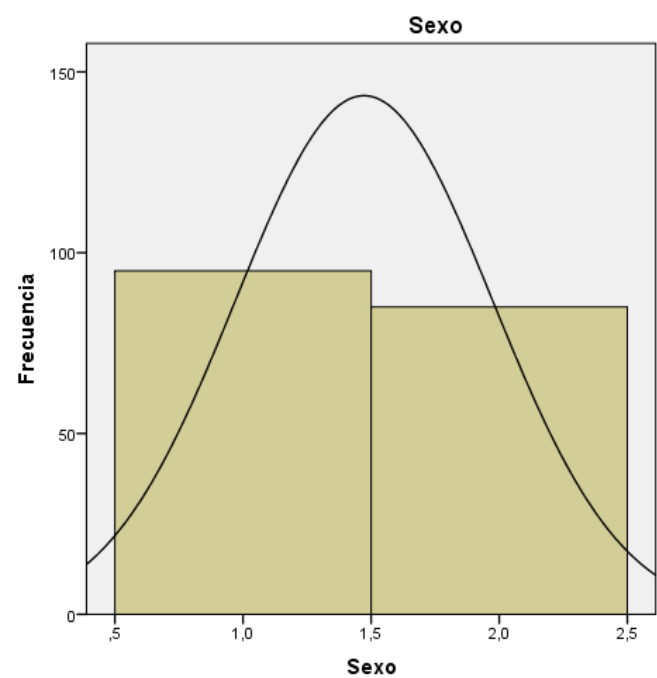

Representación gráfica de la variable "sexo" en el estudio

FIGURA 2 GRÁFICO DE LA VARIABLE INTERVINIENTE "DIAGNÓSTICO ANATOMOPATOLÓGICO"

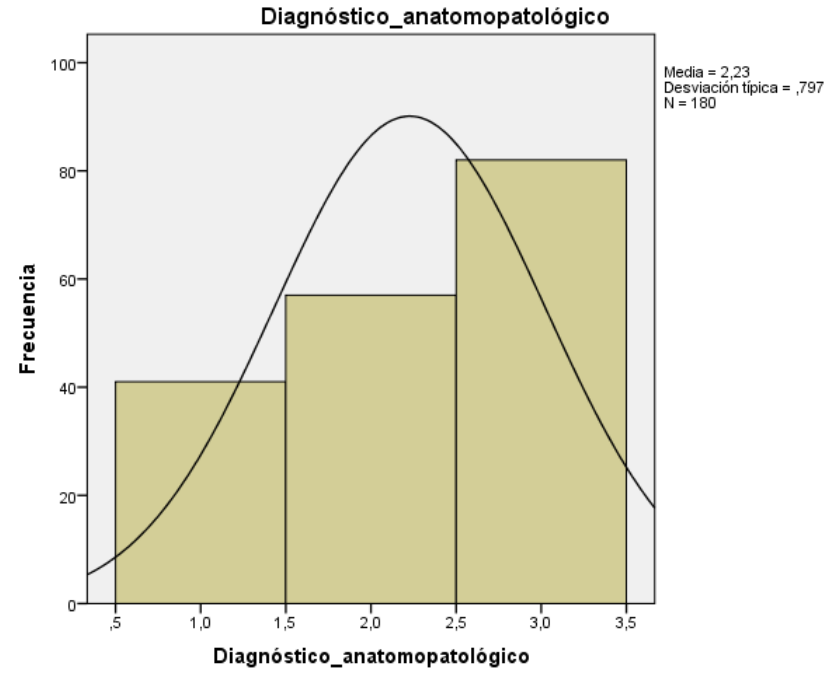

Representación gráfica de la variable "diagnóstico anatomopatológico" en el estudio

En cuanto a los resultados obtenidos relacionados con el nivel de dependencia observamos en la escala de Lawton y Brody, una puntuación media de 4,31 $( \pm 1,832)$ puntos, lo que se identifica como unos niveles de dependencia moderados; mientras que, si atendemos a la evaluación de las actividades básicas de la vida diaria, medidas con el índice de Barthel, tenemos una puntuación media de $42,53( \pm 2,789)$ puntos, es decir un nivel de dependencia moderado (Tabla 3). Siendo los individuos con diagnóstico anatomopatológico de pulmón los que presentan peores puntuaciones.

TABLA 3 ANÁLISIS DESCRIPTIVO DE LAS VARIABLES A ESTUDIO

\begin{tabular}{c|c|c|c|}
\hline \multicolumn{2}{c|}{ ÍNDICE BARTHEL } & \multicolumn{2}{c|}{$\begin{array}{c}\text { ESCALA } \\
\text { LAWTON_BRODY }\end{array}$} \\
\hline MEDIA & $\mathrm{S}$ & MEDIA & $\mathrm{S}$ \\
\hline 42,53 & 2,789 & 4,31 & 1,832 \\
\hline $\begin{array}{l}\text { NIVEL DEPENDENCIA } \\
\text { MODERADO }(A B V D)\end{array}$ & $\begin{array}{c}\text { NIVEL DEPENDENCIA } \\
\text { MODERADO }(\text { AIVD })\end{array}$ \\
\hline
\end{tabular}

Resultados descriptivos variables a estudio

En cuanto a las correlaciones, se observa, por una parte, una correlación fuerte y estadísticamente significativa entre las puntuaciones de la escala Lawton y Brody $y$ las del índice de Barthel $(r=0,803$, para $\mathrm{p}<0,05)$, por otra, obtuvimos una correlación significativa $(r=-0,403$, para $\mathrm{p}<0,001)$ entre la edad y la realización de actividades instrumentales de la vida diaria, a mayor edad se correlaciona con mayor nivel de dependencia de los individuos (Tabla 4).

TABLA 4 ESTUDIO ANALÍTICO DE LAS CORRELACIONES

\begin{tabular}{c|c}
\hline CORRELACIONES & LAWTON BRODY \\
\hline BARTHEL & $\mathrm{r}=0,803(\mathrm{p}<0,05)^{*}$ \\
\hline EDAD & $\mathrm{r}=-0,403(\mathrm{p}<0,001)^{*}$ \\
\hline
\end{tabular}

*Correlación de Pearson

Estudio de las correlaciones entre variables a estudio

Por el contrario, no se puede establecer ninguna relación significativa entre el género de los individuos y los niveles de dependencia expresados. 


\section{Discusión}

El objetivo principal del estudio fue evaluar el nivel de dependencia de los/las pacientes oncológicos/as en tratamiento activo con sintomatología respiratoria. Se buscó conocer con mayor exactitud cuál era la situación actual de las personas que se encuentran en tratamiento activo.

En los últimos años siendo conocedores del importante aumento que ha experimentado la supervivencia en las personas con $\mathrm{Ca}$, desde el año 1990 ha aumentado un $20 \%$ la supervivencia a los 5 años (Williams, 2015), es necesario conocer lo que va ligado a ese aumento de supervivencia en cuanto a la calidad de vida relacionada a la salud de estos. De acuerdo con la literatura científica se observa como uno de los mayores y más precisos indicadores de esta calidad de vida, es el nivel de dependencia que expresan los/las pacientes. Esto se relaciona directamente con lo expresado en estudios de autores como Fernández y colaboradores, el cual expone la problemática existente en términos de dependencia en pacientes oncológicos con sintomatología de tipo respiratoria, analizando en un estudio con una muestra final de 113 individuos, procedentes de la Unidad de Oncología del Complejo Hospitalario Universitario de Salamanca graves incidencias en los niveles de funcionalidad íntimamente relacionadas con los niveles de disnea. Además de incluir estudios en calidad de vida relacionada con la salud, encontrando correlaciones fuertes entre estos tres parámetros. Además de estudios de otros autores como Vockins o Purcell y colaboradores, los cuales estudiaron la afectación en términos de dependencia de los pacientes oncológicos para posteriormente proponer medidas de tipo educativo para intentar mitigar el efecto en la autonomía de los individuos (Vockins, 2004; Purcell, 2010; Fernández, 2020).

Además, no solo el aumento de la supervivencia puede condicionar o puede contribuir a un cambio en la autovalía de estas personas, sino que en muchos casos el aumento de líneas de tratamiento está demostrado que puede conllevar efectos secundarios como la astenia tumoral, el chemobrain o los problemas respiratorios (Ross, 2012; Halkett, 2005). La literatura científica los considera como uno de los mayores culpables de deterioro funcional de los individuos a estos últimos, en estudios de autores como Kealey o Holm y colaboradores (Kealey, 2005; Holm, 2012).
Otro aspecto por considerar sería la edad de los pacientes, ya que hay un claro consenso sobre la repercusión negativa que tiene la edad en términos de pérdida de autonomía. Quisimos comprobar si esto también afectaba en los/las pacientes oncológicos/as y observamos como existía una correlación estadísticamente significativa, con un grado de significación $\mathrm{p}<0,001$ si hablamos de realización de AIVD. Mientras que, en el caso de las ABVD, no podemos establecer una relación directa, en las AIVD, el grado de afectación es mayor en esta tipología de pacientes que en la normalidad de personas sin patologías oncológicas. Además, creemos oportuno continuar estudiando y profundizando sobre el tema, ya que se pueden extraer más conclusiones partiendo del estudio de personas con un estadio más avanzado, incluso partiendo del estudio de personas con cuidados paliativos.

Por otra parte, el estudio concluyó que no existe diferenciación en cuanto al género de los individuos y su capacidad funcional, no observando diferencias significativas entre hombres y mujeres en términos de pérdida de autonomía.

Consideramos importante en estudios futuros, cuantificar con mayor exactitud, una vez hemos conocido que hay un deterioro clínico en los/las pacientes oncológicos/as con patología respiratoria, la importancia que tiene ese deterioro clínico respiratorio inducido por la patología oncológica o por sus tratamientos en la pérdida de autonomía de las personas. Cuantificando el grado de afectación respiratoria y correlacionándolo con la pérdida funcional, para poder conocer todo con mayor exactitud.

En este sentido, creemos importante poder incluir la figura del terapeuta ocupacional para la atención de las personas desde un abordaje de tipo rehabilitador, gracias al cual se pueda contener el deterioro clínico funcional. Considerando importante, no solo, la intervención hospitalaria, sino un posterior seguimiento en domicilio para poder extrapolar todo lo aprendido en un contexto real. Estudios de Pergolotti o Fernández y colaboradores sobre población oncológica con sintomatología como la astenia tumoral, la disnea o el daño cerebral adquirido, como consecuencia del proceso tumoral, avalan estas afirmaciones e invitan a introducir de manera sistemática la figura del terapeuta ocupacional en la intervención del paciente con cáncer. 


\section{Conclusiones}

- Los/las pacientes oncológicos/as en tratamiento activo presentan unos niveles de dependencia moderada en la realización de sus actividades de la vida diaria.

- Correlación fuerte entre los niveles de realización de actividades instrumentales y básicas de la vida diaria en pacientes oncológicos/as en tratamiento activo.

- Pacientes oncológicos/as con mayor edad presentan mayores niveles de dependencia.

- No existe relación entre el género de los/las pacientes con Ca y la realización de actividades instrumentales de la vida diaria.

- Los/las pacientes oncológicos con un diagnóstico anatomopatológico de Ca pulmonar presentaron los peores niveles de dependencia.

\section{Aspectos Éticos del Estudio}

Para la realización del estudio, previo al inicio de este, se obtuvo permiso por parte del comité de bioética de la Universidad de Salamanca.

Todos/as los/as participantes firmaron el consentimiento informado, autorizando su participación en el mismo. $\mathrm{Y}$ a todos/as ellos/as se les explicó todo lo relacionado con el mismo, incluida la posible publicación de los datos obtenidos.

\section{FUENTE DE FINANCIACIÓN}

No hubo ninguna fuente de financiación externa para la realización de este estudio.

\section{AgradeCimientos}

El estudio pudo realizarse gracias a la participación voluntaria de todos los/las pacientes, así como a la colaboración de todo el personal del Servicio de Oncología Médica del Hospital Universitario de Salamanca, España.

\section{ReferenCiAs BibliográficAS}

American Occupational Therapy Association. Standards of practice for occupational therapy. Available at http://ajot.aota.org/ article.aspx? articleid=2477354. Accessed December 15, 2015. Es necesario revisar este link, posee error.

Cooper, J. (2007). Challenges faced by occupational therapist in oncology and palliative care. En: Cooper J. Occupational therapy in oncology and palliative care. $2^{\text {a }}$ edición. Londres: Jhon Wiley, 11-27.

Curt, G.A., Breitbart, W., Cella, D. et al. (2000). Impact of cancer-related fatigue on the lives of patients: New findings from the Fatigue Coalition. The Oncologist, 5:353-360. https://www.ncbi.nlm.nih. gov/pubmed/11040270fecha acceso?

Fernandez EJ, Rihuete MI, Cruz JJ. (2018). Estudio aleatorizado sobre la influencia de un programa de rehabilitación integral functional en el control de la astenia relacionada con la enfermedad oncológica. Medicina paliativa. 25(3). 160-67.

Fernandez EJ, Sanchez C. (2020). The relation of muskuloeskeletal pain cancer-related fatigue and Kinesiophobia in oncological patients. EC Orthopaedics. 11(6); 15-21.

Fernandez EjJ, Rihuete MI, Cruz JJ. (2020). Impacto de un programa de rehabilitación integral functional en la calidad de vida del paciente oncológico con disnea. Medicina clínica. 155(5); 220-24.

Halkett, G.K., Ciccarelli, M., Keesing, S. et al. (2010). Occupational therapy in palliative care: Is it underutilized in Western Australia? Aust OccupTher J, 57:301-309. https://doi.org/10.1111 /j.1440-1630.2009.00843.xfecha acceso?

Hewitt, M., Rowland, J.H., Yancik, R. (2003). Cancer survivors in the United States: Age, health, and disability. J GerontolA Biol SciMed Sci, 58: 82-91. https://www.ncbi.nlm.nih.gov/pubmed/12560417fecha acceso?

Holm, L.V., Hansen, D.G., Johansen, C. et al. (2012). Participation in cancer rehabilitation and unmet needs: A population-based cohort study. Support Care Cancer, 20:2913-2924. https://doi. org/10.1007/s00520-012-1420-0fecha acceso

Horneber, M., Fischer, I., Dimeo, F., Ulrich, J. \& Weiss, J. (2012). Cancer related fatigue: epidemiology, pathogenesis, diagnosis and treatment. DtschArztebl Int, 109(9): 161-72.

Kealey, P., McIntyre, I. (2005). An evaluation of the domiciliary occupational therapy service in palliative cancer care in a community trust: A patient and carers perspective. Eur J Cancer Care (Engl), 14: 232-243. https://doi.org/10.1111/j.1365-2354.2005.00559. xfecha acceso?

National Comprehensive Cancer Network. Clinical Practice Guidelines in Oncology: Cancer-related-fatigue. Version 1.2018 [En línea] 2018 [Acceso 7 de Noviembre de 2018]. Disponible en: http:// www.nccn.org/professionals/physician_gls/pdf/fatigue.pdf

Pergolotti M, Cutchin M, Weinberger M, Meyer A. (2014). Occupational Therapy use by older adults with cancer. Am J Occup Ther. 68(5): 597-607. 
Pergolotti M, Deal A, Williams G, Bryant A, Reeve B, Muss H. (2015). A randomized controlled trial of outpatien cáncer rehabilitation for older adults: The CARE program. Contemp Clin Trials. 44: 89-94.

Portela MA, Sanz A, Martínez M, Centeno C. (2011). Astenia en cáncer avanzado y uso de psicoestimulantes. Astenia en cáncer avanzado y uso de psicoestimulantes. Anales del Sistema Sanitario de Navarra. Departamento de Salud, Gobierno de Navarra. 1; 471-79.

Purcell, A., Fleming, J., Bennett, S. et al. (2010). Development of an educational intervention for cancer related fatigue. BrJ OccupTher, 73:327-333. https://dx.doi.org/10.1186\%2F1472-6955-7-12fecha acceso?

Ross, L., Petersen, M.A., Johnsen, A.T. et al. (2012). Are different groups of cancer patients offered rehabilitation to the same extent? A report from the population-based study "The Cancer Patient's World". Support Care Cancer, 20:1089-1100. https://doi.org/10.1007/ s00520-011-1189-6fecha acceso?

Sánchez, F., Barco, E. (2008). Cuidados continuos en oncología (IV): control de síntomas más prevalentes: emesis y otras complicaciones digestivas, astenia, caquexia, otros síntomas. Cuidados al final de la vida. El duelo. En: Cruz JJ, Rodríguez CA, Barco E. Oncología clínica. 4 a edición. Madrid: Nova Sidonia, 299-315.

Vockins, H. (2004). Occupational therapy intervention with patients with breast cancer: A survey. Eur J Cancer Care (Engl), 13:45-52. https://www.ncbi.nlm.nih.gov/pubmed/14961775fecha acceso?

Williams, G.R., Deal, A.M., Nyrop, K.A. et al. (2015). Geriatric assessment as an aide to understanding falls in older adults with cancer. Support Care Cancer, 23: 2273-2280. https://doi.org/10.1007/ s00520-014-2598-0fechaacceso? 
